\title{
A rare survival case of blunt left ventricular rupture caused by a low-energy pedestrian collision with a stationary forklift: a case report
}

\author{
Huangkai Zhu ${ }^{1,2 \#}$, Chenxu Zhang ${ }^{1,2 \#}$, Weidi Zhao ${ }^{1,2 \#}$, Xiang Xu' ${ }^{1}$, Yiting Shi ${ }^{1}$, Guofang Zhao ${ }^{1,2,3}$ \\ ${ }^{1}$ Department of Cardiothoracic Surgery, Hwa Mei Hospital, University of Chinese Academy of Sciences (Ningbo No. 2 Hospital), Ningbo, China; \\ ${ }^{2}$ Medical School of Ningbo University, Ningbo, China; ${ }^{3}$ Ningbo Institute of Life and Health Industry, University of Chinese Academy of Sciences, \\ Ningbo, China \\ \#These authors contributed equally to this work. \\ Correspondence to: Guofang Zhao. Department of Cardiothoracic Surgery, Hwa Mei Hospital, University of Chinese Academy of Sciences (Ningbo \\ No. 2 Hospital), Ningbo, China. Email: guofzhao@hotmail.com.
}

\begin{abstract}
Blunt cardiac rupture (BCR) is a rare injury with a high mortality rate. It is usually caused by high-energy traumatic accidents, such as motor vehicle collisions. For the first time, we report a rare case of BCR caused by a pedestrian collision with a stationary motor vehicle, which is a low-energy traumatic accident. This is also the first surgical survival BCR case to be reported of a contralateral ventricular rupture at the direct stress site. A 45-year-old formerly healthy Chinese woman, with no family history of heart disease, was walking in a hurry when she accidentally hit a forklift that was parked on the side of the road. The patient gradually lost consciousness, and was admitted to Hwa Mei Hospital Emergency Center 1 hour later. An ultrasound revealed a pericardial effusion about $1 \mathrm{~cm}$ deep and a small amount of peritoneal -35 effusion. Emergency computed tomography (CT) scans revealed a small amount of fluid accumulation in the right thoracic cavity, fractures of the 5 th and 6 th ribs on the right side, and pericardial effusion. The patient's blood pressure remained unstable after 1 hour of endotracheal intubation, B-ultrasound-guided pericardiocentesis, and antishock therapy; thus, open-heart surgery was deemed necessary. A large amount of blood accumulation was found in the intact pericardium. There was a small blood clot at the apex of the left ventricle near the interventricular septum. The removal of the clot revealed a tear about $1 \mathrm{~cm}$ in diameter. The patient's BCR was successfully repaired in the surgery. By the end of the 18-month follow-up period, the patient was found to have recovered well without significant complications. The internal mechanism of the case report was deceleration. Prompt diagnosis and emergency thoracotomy when BCR is suspected are key to rescuing patients, regardless of whether the accident is high energy or low energy, or if there is evidence of a direct force acting on the precordium, or the presence of pericardial rupture.
\end{abstract}

Keywords: Blunt cardiac injury (BCI); blunt cardiac rupture (BCR); thoracotomy; cardiac tamponade; case report

Submitted Jun 04, 2021. Accepted for publication Jun 21, 2021.

doi: $10.21037 / \mathrm{atm}-21-3031$

View this article at: https://dx.doi.org/10.21037/atm-21-3031

\section{Introduction}

Blunt cardiac injury (BCI) was first reported by Oluff Borch in 1676; however, it was not until three centuries later $(1,2)$ that its surgical repair became successful. BCI can present as asymptomatic transient arrhythmia or a fatal blunt cardiac rupture (BCR) (3). BCR accounts for approximately $0.045 \%$ of blunt traumas, but its mortality rate is as high as $89.2 \%$, and most patients lose their lives before reaching hospital $(4,5)$. BCI most frequently occurs in motor vehicle crashes, followed by motor vehicle collisions $(5,6)$. The severity of $\mathrm{BCI}$ and its underlying mechanisms vary depending on the type of blunt trauma. A small number of BCR cases caused 

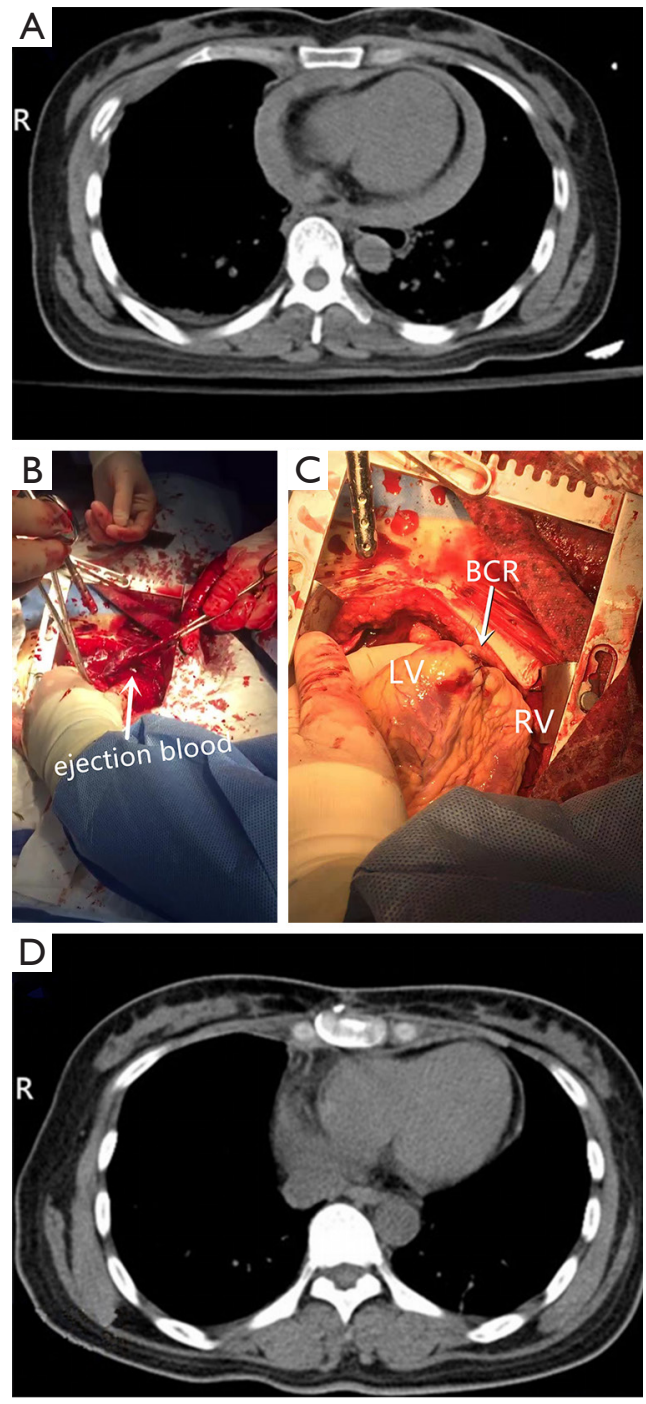

Figure 1 Chest CT scans and Operative procedure: (A) preoperatively; (B) a steady and rapid spurt of blood through the tear; (C) the tear after it was closed; (D) 2 months after the thoracotomy. CT, computed tomography; LV, left ventricle; RV, right ventricle; BCR, blunt cardiac rupture.

by low-energy traumatic accidents have been reported in previous articles; however, to date, no survival case in which the position of left ventricular rupture is different from that of force have been reported $(7,8)$. For the first time, we report a rare case of BCR caused by a pedestrian collision with a stationary motor vehicle. The patient's blunt left ventricular rupture was caused by her right lower chest colliding with a stationary forklift. The rupture was successfully repaired by surgery. We present the following article in accordance with the CARE reporting checklist (available at https://dx.doi.org/10.21037/atm-21-3031).

\section{Case presentation}

A 45-year-old formerly healthy Chinese woman, with no family history of heart disease, was walking in a hurry in a rainstorm when she accidentally hit a forklift that was parked on the side of the road. After her right lower chest collided vertically with the side of the forklift, the patient gradually lost consciousness. She was admitted to the Hwa Mei Hospital Emergency Center 1 hour later with a Glasgow Coma score of 11. Her blood pressure was undetectable, her radial pulse was weak, her heart rate was $130 \mathrm{bpm}$ (sinus rhythm), and there was no significant haemorrhagic spots or ecchymosis on the body except for the right lower chest wall. The results of the arterial blood gas analysis were as follows: total haemoglobin level, $12.3 \mathrm{~g} / \mathrm{dL}$; potential of hydrogen 7.04; partial pressure of carbon dioxide, $57 \mathrm{mmHg}$; partial pressure of oxygen, $16 \mathrm{mmHg}$; actual bicarbonate, $15.4 \mathrm{mmol} / \mathrm{L}$; standard bicarbonate, $10.8 \mathrm{mmol} / \mathrm{L}$; blood oxygen saturation, $9 \%$; and troponin I test results, $0.5 \mathrm{ng} / \mathrm{mL}$.

Based only on the clinical manifestations and the abovementioned test results, we could not confirm that the low-energy trauma accident had caused severe BCI. An ultrasound revealed a pericardial effusion about $1 \mathrm{~cm}$ deep and a small amount of peritoneal effusion. Emergency computed tomography (CT) scans revealed a small amount of fluid accumulation in the right thoracic cavity, fractures of the 5th and 6th ribs on the right side, and pericardial effusion (see Figure 1A). Based on the examination results above, we made a provisional diagnosis of BCR, cardiac tamponade, type II respiratory failure, and metabolic acidosis with multiple traumas. The patient's blood pressure remained unstable after 1 hour of endotracheal intubation, B-ultrasound-guided pericardiocentesis, and antishock therapy; thus, open-heart surgery was deemed necessary.

The patient was placed in the supine position, and the sternum was split in the middle. A hematoma was found in the mediastinum, and a large amount of blood accumulation was found in the pericardium. There was a small blood clot at the apex of the left ventricle near the interventricular septum. The removal of the clot revealed a tear about $1 \mathrm{~cm}$ in diameter. There was a steady and rapid spurt of blood through the tear (see Figure 1B). A 4-0 prolene silk suture was used in a continuous manner to close the tear (see Figure 1C). Myocardial contusion was found near 
the rupture with a range of about $2.0 \times 2.0 \mathrm{~cm}$. There was a single fracture at the front axillary line of the right 5 th and 6th ribs, and a parietal thoracic model hematoma was found to have formed. After the placement of 1 mediastinal drainage tube and 1 thoracic drainage tube, the chest was closed layer by layer. The total intraoperative blood loss was about 2,000 $\mathrm{mL}$. The patient was admitted to the intensive care unit (ICU) after surgery.

The patient's hemodynamic status was stable postoperatively, and mechanical ventilation was used. After 40 hours, the patient regained consciousness and the endotracheal intubation was removed. Four days later, the patient was transferred from the ICU to the general ward, and discharged after 18 days. The pericardial effusion almost disappeared 2 months after the thoracotomy (see Figure 1D). By the end of the 18-month follow-up period, the patient was found to have recovered well without significant complications. All procedures performed in studies involving human participants were in accordance with the Helsinki Declaration (as revised in 2013). This study was approved by the Hwa Mei Hospital, University of Chinese Academy of Sciences (Ningbo No. 2 Hospital). Written informed consent was obtained from the patient for publication of this case report and any accompanying images.

\section{Discussion}

Severe BCI, including BCR, occurs most frequently in vehicle collisions $(50 \%)$, followed by pedestrian collisions (35\%), motorcycle crashes (9\%), and falls (6\%) (3). The possible mechanisms of BCR include rib fractures, compression of heart between the sternum and vertebral column, deceleration and transmission of venous pressure due to compression of abdomen or lower extremities $(1,2,5)$. This is the first reported case of BCR caused by a pedestrian striking a stationary vehicle. Some aspects of this case should be noted. First, compared to BCI caused by high-energy trauma accidents, such as motor vehicle collisions or vehicle collisions with pedestrians, the force suffered by the patient in this case was relatively small; thus, the degree of trauma induced may not necessarily be consistent with the severity of the BCI. Similar rare lowenergy collision cases support this conclusion $(7,8)$. In 2019, Akishima et al. reported a 17-year-old boy who was accidentally kicked on the lateral side of the right chest during soccer and sustained longitudinal cardiac rupture on the right ventricular antero-inferior wall which was successfully repaired, from compression between the sternum or the costal cartilages and spine, or significant elevation of venous pressure (8). And in 2020, Singh et al. reported a 46-year-old male construction worker who fell forward onto a metal rod protruding from the ground at a constructions site and sustained left ventricular injury which was successfully repaired from a displaced rib fracture which was successfully repaired (7). However, the patient suffered from fractures in this case, its internal mechanism might be different from other low-energy collision cases. In this instance, the collision site was in the front of the right lower thoracic axillary opposite the left ventricular rupture. Thus, the internal mechanism of the left ventricular rupture could have been induced by the contre-coup injury; that is, the mechanism of the deceleration force. Such that when the patient's right lower chest collided with the forklift, the left ventricle ruptured due to the contre-coup injury caused by the collision between the heart and the sternum or the left rib due to inertia. At the same time, the risk of myocardial rupture was aggravated by the forward impact of blood on the left ventricle due to inertia.

It has been more than six decades since the first reported successful treatment of BCR in 1955, but little progress has been made in defining nomenclature and treatment guidelines for the same. Despite the benefit of early suspicion, it is only effective when coupled with the most inclusive and comprehensive modalities of diagnosis and management. Medical history, symptoms and signs, electrocardiogram examination, myocardial injury marker examination, ultrasound examination, chest X-ray, CT, etc. are all significantly helpful for early diagnosis of BCR (9). In the absence of specialized imaging examinations or other examinations, health care providers should use known indicators for comprehensive analysis to make timely judgments. In general, BCR caused by an indirect mechanism is more insidious than BCR caused by an direct mechanism and prone to missed diagnoses and misdiagnoses, which can result in delayed treatment opportunities and lead to the tragic deaths of patients. Approximately $42 \%$ of patients with BCR who are sent to hospital die in the emergency room; however, early recognition and diagnosis, followed with urgent treatment by following the advanced trauma resuscitation guidelines included maintain the level of injury and prevent deterioration, aggressive resuscitation, or surgical intervention are critical factors and younger, lower Injury Severity Score, higher Glasgow Coma Scale, an systolic blood pressure $\geq 90 \mathrm{mmHg}$, lower preoperative creatinine 
level, lower creatine kinase-myocardial band level and lower platelet count are possible factors for survival and recovery of the BCR patients with or without other lifethreatening complex injuries $(5,10,11)$.

In this case, the patient's pericardium was intact; however, pericardial rupture has been found to occur in $30 \%$ of severe BCI cases (4). Thus, the possibility of cardiac rupture should also be considered in trauma patients with pleural haematoma with little pericardial effusion (12). Pericardiocentesis was performed preoperatively to alleviate the symptoms of acute tamponade at the time, but the patient's symptoms did not improve significantly. Consequently, an emergency thoracotomy was immediately performed to repair the rupture. In the instances where noninvasive intervention is ineffective in stabilizing the patient, surgical intervention "thoracotomy", namely a lifesaving procedure, is highly and promptly recommended, to mitigate distress in cases such as hemorrhage control, and tamponade, internal cardiac massage (9). Of the patients surviving to operation, $42 \%$ survived $>24$ hours of which $87 \%$ were discharged (5). Rare cases of delayed BCR have been reported $(13,14)$. Thus, it is necessary to carefully examine the possibility of rupture of other parts of the heart during the operation and conduct long-term follow-up examinations.

This case had a number of limitations. The patient stated that she had no cardiovascular disease; however, no recent cardiac B-ultrasound examination results were available to prove this. In addition, the patient received a thoracotomy, as she experienced a pericardial tamponade immediately after the left ventricular rupture, which resulted in a decrease in blood pressure and the reduction of bleeding from the rupture, and thus the formation of a blood clot. As the blood clot blocked the ventricular rupture, the severity of the pericardial tamponade did not increase. Before the operation, we did not consider the risk of the shedding of the blood clot; thus, no corresponding prevention measures were formulated, especially during pericardiocentesis and patient transport. Fortunately, the shedding of the blood clot did not occur.

\section{Conclusions}

Prompt diagnosis and emergency thoracotomy when BCR is suspected are key to rescuing patients, regardless of whether the accident is high energy or low energy, or if there is evidence of a direct force acting on the precordium, or the presence of pericardial rupture.

\section{Acknowledgments}

Funding: This study was supported by the Medical Health Science and Technology Project of Zhejiang Provincial Health Commission (Grant No. 2021430921).

\section{Footnote}

Reporting Checklist: The authors have completed the CARE reporting checklist. Available at https://dx.doi. org/10.21037/atm-21-3031

Conflicts of Interest: All authors have completed the ICMJE uniform disclosure form (available at https://dx.doi. org/10.21037/atm-21-3031). The authors have no conflicts of interest to declare.

Ethical Statement: The authors are accountable for all aspects of the work in ensuring that questions related to the accuracy or integrity of any part of the work are appropriately investigated and resolved. All procedures performed in studies involving human participants were in accordance with the Helsinki Declaration (as revised in 2013). This study was approved by the Hwa Mei Hospital, University of Chinese Academy of Sciences (Ningbo No. 2 Hospital). Written informed consent was obtained from the patient for publication of this case report and any accompanying images.

Open Access Statement: This is an Open Access article distributed in accordance with the Creative Commons Attribution-NonCommercial-NoDerivs 4.0 International License (CC BY-NC-ND 4.0), which permits the noncommercial replication and distribution of the article with the strict proviso that no changes or edits are made and the original work is properly cited (including links to both the formal publication through the relevant DOI and the license). See: https://creativecommons.org/licenses/by-nc-nd/4.0/.

\section{References}

1. Warburg E. Myocardial and pericardial lesions due to nonpenetrating injury. Br Heart J 1940;2:271-80.

2. Bellister SA, Dennis BM, Guillamondegui OD. Blunt and penetrating cardiac trauma. Surg Clin North Am 2017;97:1065-76.

3. Biondi NL, Bhandari M, Bhyan P. Transient right bundle branch block resulting from a blunt cardiac injury during a 
motor vehicle accident. Cureus 2020;12:e10534.

4. Baldwin D, Chow KL, Mashbari H, et al. Case reports of atrial and pericardial rupture from blunt cardiac trauma. J Cardiothorac Surg 2018;13:71.

5. Teixeira PG, Inaba K, Oncel D, et al. Blunt cardiac rupture: a 5-year NTDB analysis. J Trauma 2009;67:788-91.

6. Huis In 't Veld MA, Craft CA, et al. Blunt cardiac trauma review. Cardiol Clin 2018;36:183-91 .

7. Singh Y, Arra A, Cawich SO, et al. A case report of blunt cardiac rupture. Int J Surg Case Rep 2020;73:244-7.

8. Akishima S, Takeyasu N. Blunt cardiac rupture due to kicking on the lateral side of the right chest. Clin Case Rep 2019;7:1806-8.

9. Fadel R, El-Menyar A, ElKafrawy S, et al. Traumatic blunt cardiac injuries: an updated narrative review. Int J Crit Illn Inj Sci 2019;9:113-9.

10. Janicic D, Simatovic M, Roljic Z, et al. Urgent surgical

Cite this article as: Zhu $\mathrm{H}$, Zhang $\mathrm{C}$, Zhao W, Xu X, Shi Y, Zhao G. A rare survival case of blunt left ventricular rupture caused by a low-energy pedestrian collision with a stationary forklift: a case report. Ann Transl Med 2021;9(12):1028. doi: 10.21037/atm-21-3031 treatment of blunt chest trauma followed by cardiac and pericardial injuries. Med Arch 2020;74:115-8.

11. Yun JH, Byun JH, Kim SH, et al. Blunt traumatic cardiac rupture: single-institution experiences over 14 years. Korean J Thorac Cardiovasc Surg 2016;49:435-42.

12. Oizumi H, Suzuki K, Hoshino H, et al. A case report: hemothorax caused by rupture of the left atrial appendage. Surg Case Rep 2016;2:142.

13. Pooniya S, Behera C, Mridha AR, et al. Cardiac rupture delayed for a week in an asymptomatic child following blunt trauma. Med Sci Law 2016;56:217-20.

14. Ueda S, Ito Y, Konnai T, et al. Delayed cardiac rupture occurring two months after blunt chest trauma. Gen Thorac Cardiovasc Surg 2011;59:45-7.

(English Language Editor: L. Huleatt) 